\title{
Myrcene enhances the cardioprotective effect through matrix remodelling in an experimental model of heart failure
}

\author{
Jiawei Tian ${ }^{1}$, Rui Zhang ${ }^{2}$, Yunqi Weng ${ }^{1}$, Qiaoji Qin ${ }^{1}$, Xuezhi Zhang ${ }^{1}$, Aiguo Liu ${ }^{1}$, Nan Lin ${ }^{1}$
}

\author{
${ }^{1}$ Department of Emergency, The Affiliated Hospital of Qingdao University, Qingdao, \\ Shandong Province, China \\ 2Department of Cardiology, The Affiliated Hospital of Qingdao University, Qingdao, \\ Shandong Province, China
}

Submitted: 3 March 2020

Accepted: 1 April 2020

Arch Med Sci

DOI: https://doi.org/10.5114/aoms.2020.95875

Copyright $\odot 2020$ Termedia \& Banach

\section{Abstract}

Introduction: Myrcene, a terpene found in essential oils sourced from plants, is known to elicit various biological effects such as antioxidant, anti-inflammatory, hypoglycaemic, and hepatoprotective activities. However, reports on the protective effect of myrcene against heart failure are still not clear. Hence, in the present study, the experimental model of heart failure was induced using isoproterenol in rats.

Material and methods: Wistar male rats were divided into four groups: group I - Control; group II - ISO-induced heart failure; group III - Myrcene pre-treated (1.0 mg/kg/day and ISO); and group IV - Myrcene drug control for the experimental period of 4 weeks. At the end of the treatment, infarct size, cardiac markers, cell stress enzymes such as creatinine kinase, lactate dehydrogenase, inflammatory markers, matrix proteins, and microRNA signatures were elucidated using RT-PCR, ELISA, and immunohistochemistry analysis.

Results: Rats with induced heart failure demonstrated abnormality in cardiac functions and marker gene expressions, while myrcene pre-treated rats prevented cardiac failure significantly $(p<0.001)$ with restoration of cellular functions. Also, myrcene pre-treatment abrogated the inflammatory signals with restored matrix proteins. Furthermore, the microRNA of matrix signalling pathways were also found to be prominently reduced compared to heart failure rats.

Conclusions: Myrcene protected hearts from ISO-induced failure in rats and provided a cardioprotective effect via restoring the matrix signalling, anti-inflammatory, and antioxidant effect, which suggests a possible drug candidature for the treatment of cardiac failure.

Key words: cardioprotective, isoproterenol, myocardial infarction, oxidative stress, myrcene.

\section{Introduction}

In the industrialised world, more and more people are aged above 60 years, and hence more funds are needed for their healthcare, which is primarily affected by chronic diseases. In a way, modernised medical care facilities are improving the life of the average person. Nonetheless, aging and the associated chronic diseases reduce the productive life of the

\author{
Corresponding author: \\ Nan Lin \\ Department of Emergency \\ The Affiliated Hospital \\ of Qingdao University \\ 16 Jiangsu Road \\ Shinan District \\ Qingdao 266000 \\ Shandong Province \\ China \\ Phone/fax: +86 5328291 \\ 1283 \\ E-mail: linnqd@163.com
}


people. This is a critical feature of health because these are causes of earlier mortality and can be prevented by lifestyle measures and medicines. Hence, chronic noncommunicable diseases are gaining importance, and heart failure being the leading cause of morbidity in the aged is a prime concern for all health departments of the world.

Heart failure may be defined as the rapid onset of clinical symptoms that are presented due to abnormal systolic and diastolic function of the heart $[1,2]$. Possible features of heart failure and the reasons include reduced cardiac output, tissue hypoperfusion, an increase in pulmonary artery occlusion pressure, and congestion in the cardiac tissues [3]. Patients who suffer from acute heart failure may not have a history of cardiac dysfunction, and acute failure is associated with intense discomfort in the chest and high mortality. Urgent treatment in cases of acute cardiac failure is important [4].

Risk factors of cardiac failure include changes that are structurally and functionally affected by endothelial inflammation, which ultimately lead to atrophy of the cardiac muscles and increased cardiomyocyte stiffness. Inflammatory cytokines and biomarkers produced as a part of cardiac failure have been used for diagnosis of heart failure [5]. Aging-related complications include remodelling of the atrium, fibrosis of the cardiac muscles, and vascular inflammation. Increased oxidative damage in aging promotes proinflammatory cytokines and chemokines released from the cardiomyocytes and recruits macrophages. Matrix metalloproteinases that are produced by the macrophages favour the degradation of extracellular matrix components and increase these proteins' turnover [6-8].

Patients with heart failure express specific glycoproteins at transient levels and deposit collagen in the damaged myocardial tissues [9]. Because endothelial inflammation is an important event in the pathogenesis of cardiac failure, new treatments must include bioactive compounds that are immunomodulatory [10]. Myrcene is a naturally occurring hydrocarbon that is found in lemongrass, hops, and cannabis. It is volatile and is used in the perfume industry. It has anti-inflammatory [11], anti-bacterial, and analgesic properties [12] that are widely used in traditional medicines [13].

The present study aimed at the anti-inflammatory property of myrcene in alleviating the complications of heart failure. In the process of achieving it, rats were used and cardiac failure was induced using ISO and then treated with myrcene to reduce the effects of heart failure and related cardiac markers, expression of inflammatory cytokines, and other matrix components of the heart. Hence, we propose myrcene as a probable drug candidate in the treatment of heart failure primarily through the reduction of inflammation.

\section{Material and methods}

\section{Experimental model of heart failure}

For the present study, male Wistar rats (120 $\pm 10 \mathrm{~g})$ were used. All animals were kept in cages in a temperature-controlled room with a 12-hour light-dark cycle with temperature and humidity maintained at $25 \pm 2^{\circ} \mathrm{C}$ and $55 \pm 5 \%$, respectively. Rats received free access to regular standard rat chow and RO water. The animal experimental protocol was followed after obtaining prior permission from the Institutional Animal Ethical Committee, and the experiments were carried out strictly in accordance with the institutional guidelines and suggestions of the ethical committee. For the experimentation, eight animals per group were divided randomly and allocated to four groups as follows: Group 1 as a vehicle-control group; group 2 as an ISO-induced group (120 mg isoproterenol per $\mathrm{kg}$ body weight of the animal was administered intraperitoneally); group 3 rats received myrcene $(20 \mathrm{mg} / \mathrm{kg}$, orally) administration, which was started a week before; group 4 rats were given myrcene alone as drug controls. The rats were maintained for 8 weeks. At the end of the experimental period, the animals were assessed for the echocardiographic measures as described earlier [14]. The animals were then killed, the heart tissue was dissected, the heart weight to body weight ratio was calculated, and a portion of heart tissue was used for histopathological analysis and collagen accumulation [15].

\section{Cardiac markers and cytokine analysis}

The onset of heart failure was elucidated using the levels of markers such as CK, LDH, and troponin I. Also, levels of anti-inflammatory and pro-inflammatory markers such as interleukin (IL)-6, IL-4, tumor necrosis factor $\alpha$ (TNF- $\alpha$ ), interferon $\gamma$ (IFN- $\gamma), I L-1 \beta$, and IL-10 were elucidated by ELISA measurements using commercial assay kits as per the manufacturer's instruction.

\section{Reverse transcription-polymerase chain reaction ( $R T-P C R)$}

To evaluate the expression of microRNA in the present study, quantitative RT-PCR was performed with miR-specific primers. RNA was isolated from the heart tissues using TRIzol reagent and quantified. For quantitative detection of miR-21, miR208a, miR-29, miR-19b, and miR-133, TaqMan MicroRNA Reverse Transcription Kit, TaqMan miR specific assays, and snoRNA assays were used according to the manufacturer's instructions. SnoR- 
Table I. List of primers used in our study

\begin{tabular}{|c|c|c|c|}
\hline Gene & Primer & Sequence & Annealing \\
\hline \multirow[t]{2}{*}{ MMP-2 } & $\mathrm{F}$ & CCGTTATGAGACCCTGAGCC & 59 \\
\hline & $R$ & CAGACCAATCGTGCCTCCAT & \\
\hline \multirow[t]{2}{*}{ MMP-9 } & $\mathrm{F}$ & GATCCCCAGAGCGTTACTCG & 58 \\
\hline & $\mathrm{R}$ & GTTGTGGAAACTCACACGCC & \\
\hline \multirow[t]{2}{*}{ TGF- $\beta$} & $\mathrm{F}$ & AGGGCTACCATGCCAACTTC & 57 \\
\hline & $R$ & CCACGTAGTAGACGATGGGC & \\
\hline \multirow[t]{2}{*}{ PAI-1 } & $\mathrm{F}$ & GTGGTTCGGCACAATCCAAC & 57 \\
\hline & $\mathrm{R}$ & AAGATTTACCAGTGCCGGGG & \\
\hline \multirow[t]{2}{*}{$\alpha-S M A$} & $\mathrm{~F}$ & ACCATCGGGAATGAACGCTT & 58 \\
\hline & $\mathrm{R}$ & CTGTCAGCAATGCCTGGGTA & \\
\hline \multirow[t]{2}{*}{ Fibronectin } & $\mathrm{F}$ & TAGAGGCAGGCTAGTGTGGA & 59 \\
\hline & $\mathrm{R}$ & GAAGCCAAAGATGACCTGTGA & \\
\hline \multirow[t]{2}{*}{ CTGF } & $\mathrm{F}$ & CTTCCCGAGAAGGGTCAAGC & 58 \\
\hline & $R$ & GGCTCGCATCATAGTTGGGT & \\
\hline \multirow[t]{2}{*}{ Col3a1 } & $\mathrm{F}$ & TGCAATGTGGGACCTGGTTT & 57 \\
\hline & $\mathrm{R}$ & GGGCAGTCTAGTGGCTCATC & \\
\hline \multirow[t]{2}{*}{ GAPDH } & $\mathrm{F}$ & AGTGCCAGCCTCGTCTCATA & 59 \\
\hline & $\mathrm{R}$ & TTCTCAGCCTTGACTGTGCC & \\
\hline
\end{tabular}

NA was used as a housekeeping control for normalisation.

To further elucidate the mRNA expression of fibrotic markers, a known amount of RNA sample was transcribed to cDNA, and the real-time PCR was done for specific genes using $S_{Y B R}{ }^{\circledR}$ Green/ROX master mix (Thermo Fisher Scientific, Waltham, MA). The gene-specific primers used in the study are listed in the Table I. The obtained $C t$ values were used for the calculation of gene expressions by the comparative $C t$ method $(\Delta \Delta C t)$ using GAPDH as the housekeeping gene.

\section{Immunohistochemical analysis}

Briefly, $5 \mu \mathrm{m}$ paraffin sections of the heart tissues were rehydrated, and the antigen was retrieved using citrate buffer ( $\mathrm{pH}$ 6.0). Later, the nonspecific binding in the tissue sections was blocked using 3\% BSA in PBS. Then the antigen-antibody reactions were carried out on the tissue sections by incubating with iNOS- and TGF- $\beta$-specific primary polyclonal antibody $(1: 200)$ diluted in $1 \%$ skimmed milk powder in PBS for $1.5 \mathrm{~h}$ at room temperature, washed with PBST, and then incubated with corresponding HRP-labelled secondary antibodies for $1 \mathrm{~h}$ at room temperature. Then the antigen expression was visualised using DAB per- oxidase activity, counterstained with haematoxylin, and mounted using DPX.

\section{Statistical analysis}

Statistical significance was evaluated with a one-way analysis of variance (ANOVA) and Student's $t$-test. Data were expressed as the mean \pm SEM. Graph Pad Prism version 5.0 was used for the analyses. Differences with a $p$-value of less than 0.05 were considered statistically significant.

\section{Results}

The present study aimed at evaluating the cardioprotective mechanism of myrcene by which the rescue signalling was activated in an experimental model of heart failure and the results presented below. Figure 1 shows significant $(p<0.01)$ increases in the HW/BW ratio, levels of creatinine kinase (CK), lactate dyhydrogenase (LDH), troponin I, and modulated echocardiographic parameters in ISO-induced rats compared to controls, while rats pre-treated with myrcene displayed restored cardiac functions with decreased levels of CK, LDH, and troponin I compared to ISO-induced rats (Figure 1).

Figure 2 represents the histopathological analysis and Masson's trichrome staining performed in the heart tissue sections of control and ex- 
A

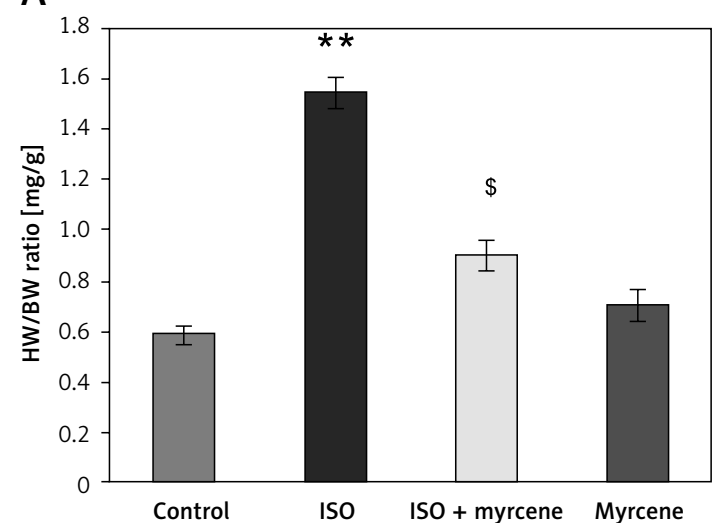

C

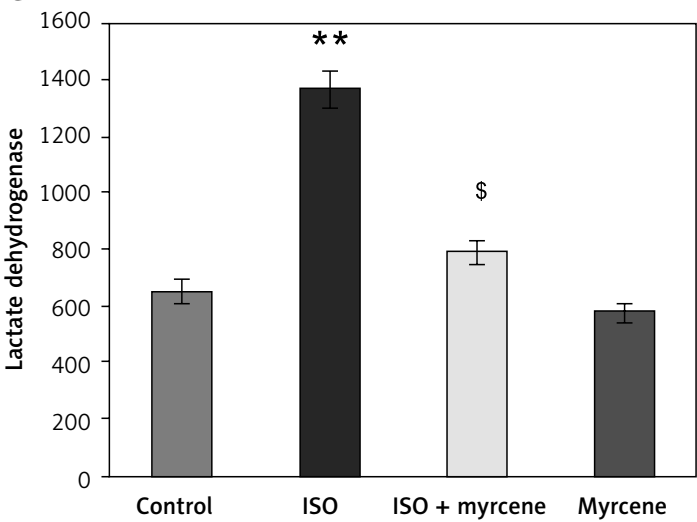

E

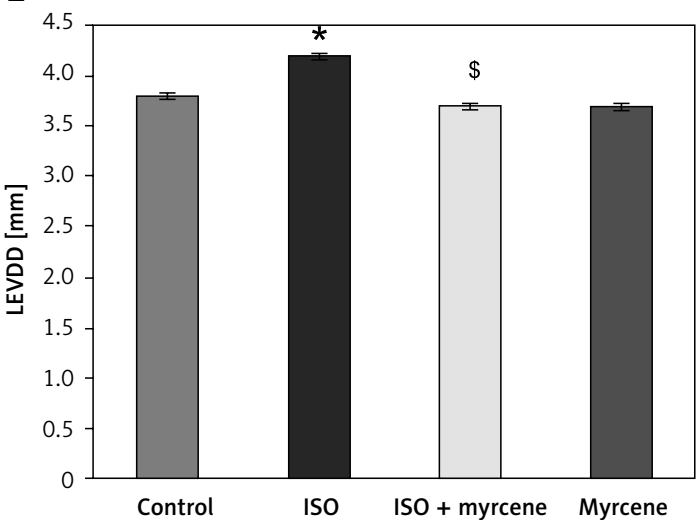

B

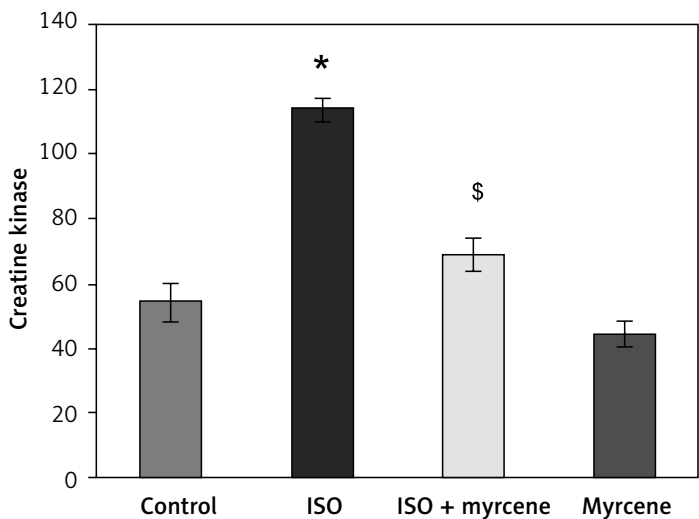

D

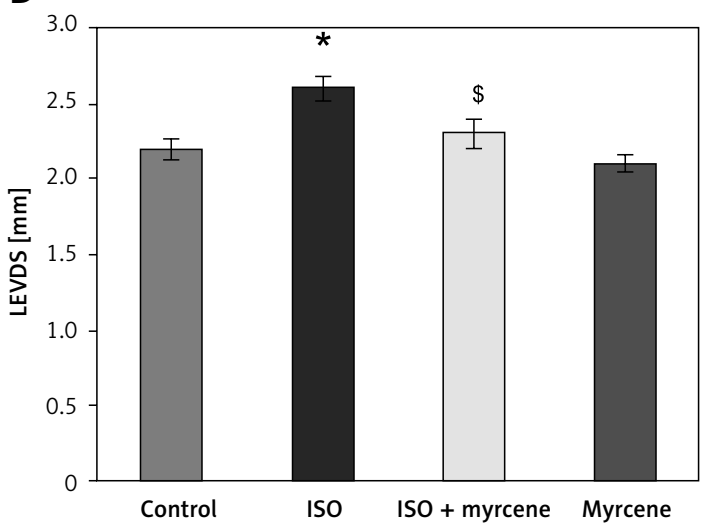

$\mathbf{F}$

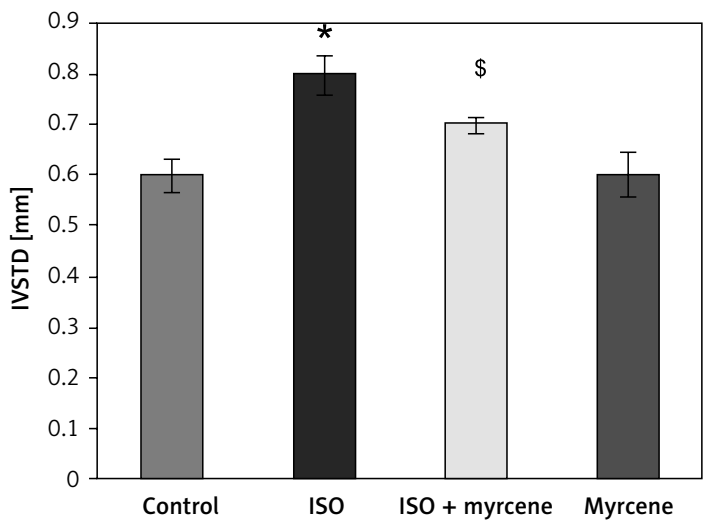

Figure 1. A-F represents the HW/BW ratio, levels of $\mathrm{CK}, \mathrm{LDH}$, and echo cardiac parameters in the control and experimental groups of rats. CK activity is expressed as $\mu \mathrm{mol}$ of phosphorus liberated/mg of protein/h. LDH activity is expressed as $\mu \mathrm{mol}$ of pyruvate liberated $/ \mathrm{mg}$ of protein $/ \mathrm{h}$

Values are expressed as mean $\pm S E(n=12)$. Statistical significance expressed as ${ }^{*} p<0.05,{ }^{* *} p<0.01$ compared to vehicletreated controls, ${ }^{s} p<0.0515 O+$ myrcene compared to ISO-induced rats.

perimental animals. Animals induced with ISO showed increased fibrosis (2.5-fold, $p<0.01)$, as shown by increased blue staining in the heart tissue sections of the ISO group. In addition, $\mathrm{H} \& \mathrm{E}$ staining also revealed the damaged tissue fibrils in ISO animals, whereas treatment with myrcene resulted in a marked reduction of collagen deposition in the heart tissues. While no significant changes were observed in the myrcene-only group compared to controls (Figure 2).
Furthermore, the cytokine levels were estimated in controls, and the levels in experimental rats are presented in Figure 3. The results show that the increased levels of cytokines such as IL-6 $(p<0.01)$, IL-4 $(p<0.05),(p<0.05)$ TNF- $\alpha(p<0.01)$, IFN- $\gamma$ $(p<0.01)$, and IL-1 $\beta(p<0.05)$ with reduced IL-10 levels were found in ISO rats compared to controls. These inflammatory cytokines were diminished in the myrcene pre-treatment with improved IL-10 levels $(p<0.01)$, showing that the protective role of myrcene 
A

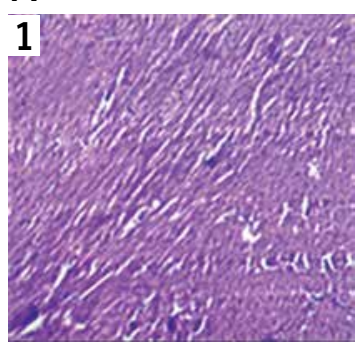

B
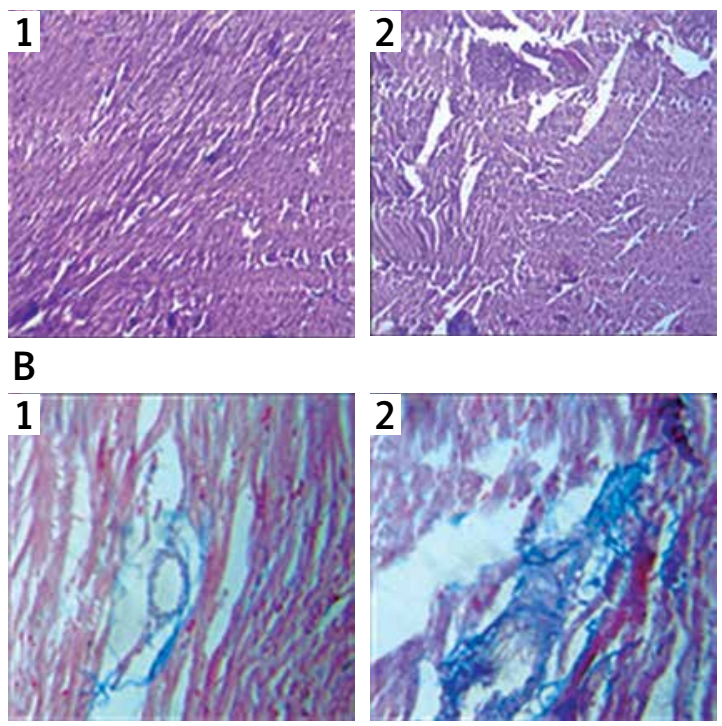

C

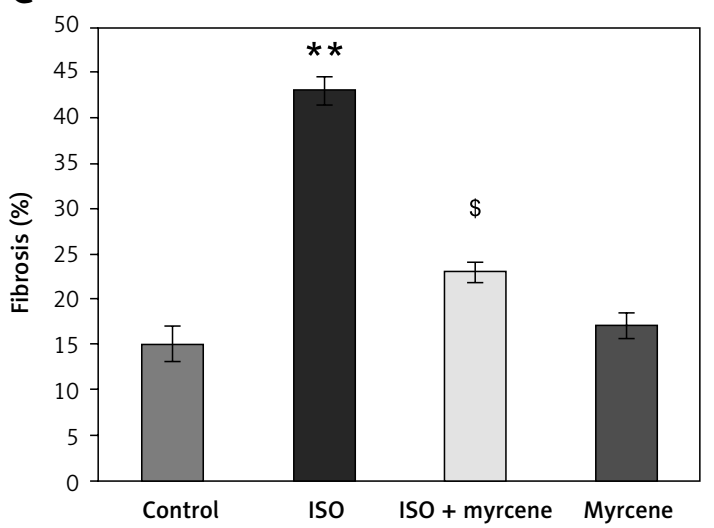

is through blocking aggravation of the inflammatory signalling in the cardiac tissues (Figure 3).

To substantiate the role of myrcene on the modulation of fibrotic markers, RT-PCR was carried out, and the results are presented in Figure 4. Rats suffering from heart failure demonstrated a significant increase in fibrotic markers such as MMP-2 (3.5-fold), MMP-9 (3-fold), TGF- $\beta$ (3.2fold), PAl-1 (2.7-fold), $\alpha$-smooth muscle action (4.2-fold), fibronectin (2.1-fold), CTGF (2.5-fold), and collagen-1 (3.2-fold) mRNA compared to controls. However, the increased levels of these genes were seen to be normalised in myrcene treatment, suggesting that the drug exerts an anti-fibrotic effect in the tissue (Figure 4).

The role of myrcene in the cardioprotective role via microRNAs was evaluated using PCR. Figure 5 shows the expression level of these microRNA, and the results demonstrated the increased level of miR-21 (2.2.-fold) and miR-208a (3.1-fold) with reduced levels of anti-fibrotic miR-29, miR-19b, and miR-133 in ISO-induced rats. While the levels of these miRs were restored $(p<0.01)$ in myrcene, pre-treatment suggests that the interplay of mi-
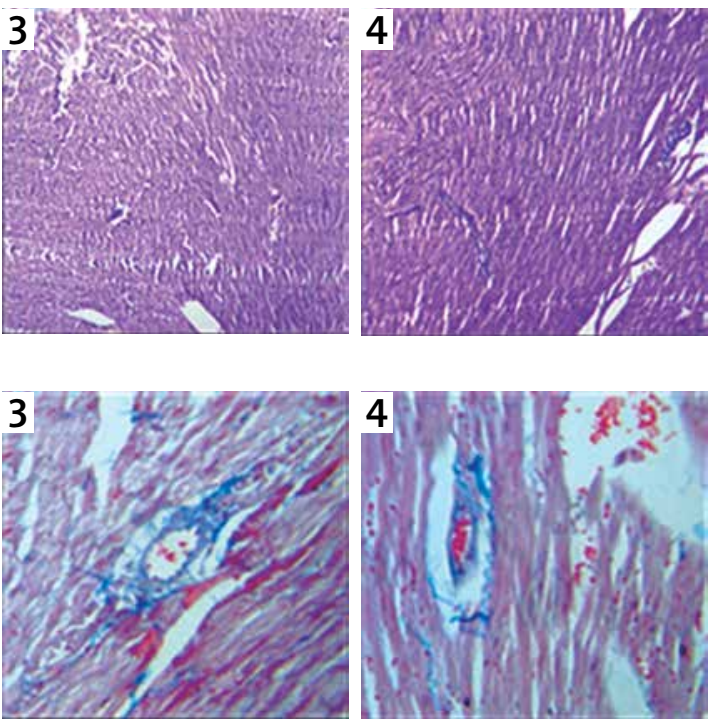

Figure 2. A-C represents the histopathological analysis and Masson's trichrome staining in the control and experimental group of rats $(\mathbf{1}-$ Control; 2 - ISO; 3 - ISO + myrcene; 4 - Myrcene). The amount of collagen deposition was compared between groups and expressed in percentage

Values are expressed as mean $\pm S E(n=12)$. Statistical significance is expressed as ${ }^{* *} p<0.01$ compared to vehicle-treated controls, ${ }^{s} p<0.051 S O+$ myrcene compared to $15 O$-induced rats.

croRNAs is one of the clues in the aggravation of heart failure (Figure 5).

Figure 6 shows the immunohistochemical (IHC) analysis in the heart tissues of control and myrcene-treated rats. In order to show the tissue level expression of the inflammatory mediator and fibrotic marker, the IHC analysis of iNOS and TGF- $\beta$ expression were elucidated in the hearts of experimental rats. The results demonstrate an increase $(p<0.05)$ in chemical reactivity for the iNOS and TGF- $\beta$ proteins in ISO-induced rats, while the levels of these proteins were attenuated in myrcene-pre-treated rats compared to the heart-failure rats. No significant changes in the expression of these proteins were detected in the heart tissue of drug control animals (Figure 6).

\section{Discussion}

In response to acute myocardial injury, the heart undergoes hypertrophy as a compensatory response, and such responses were also observed when ISO was used to induce cardiac hypertrophy [16]. In the present study, the abnormalities in the 
A

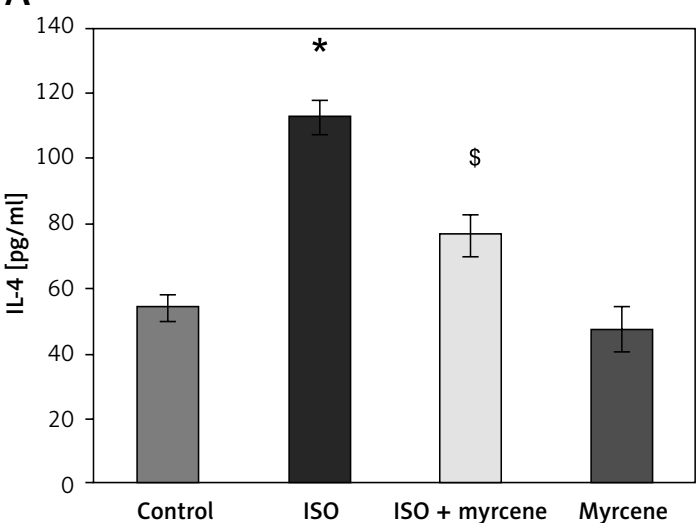

C

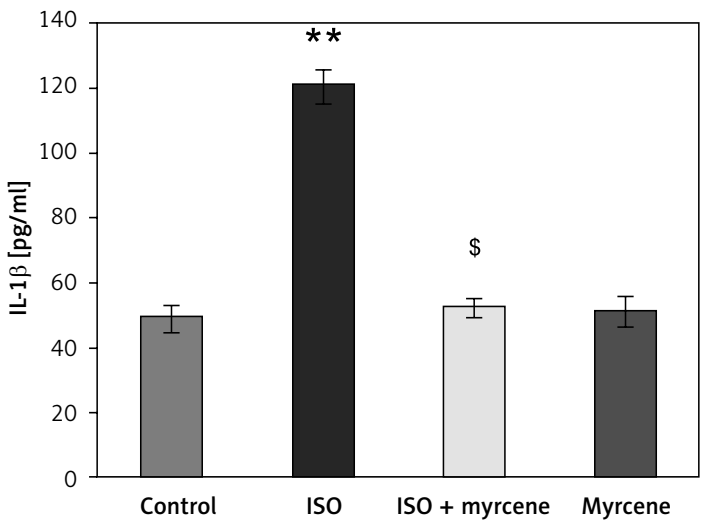

$\mathrm{E}$

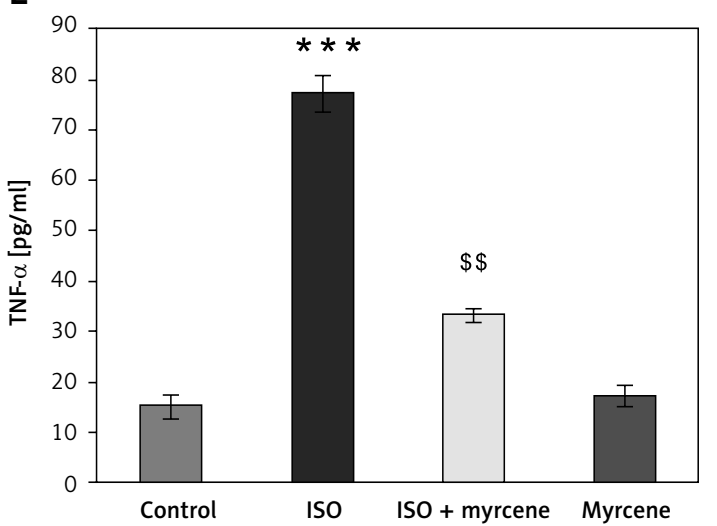

B

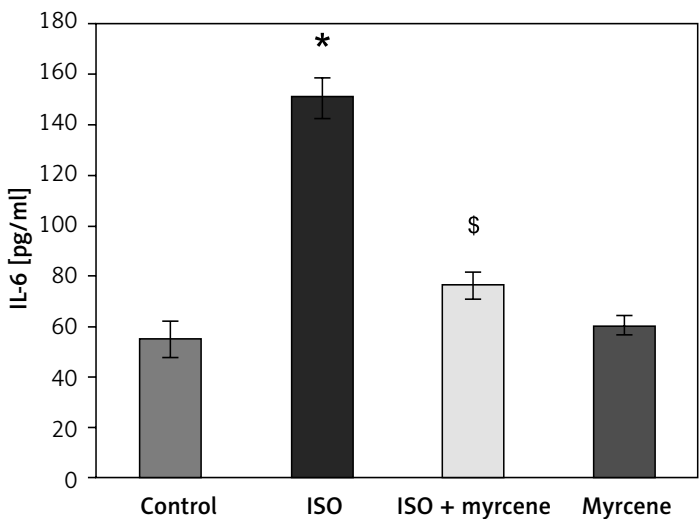

D

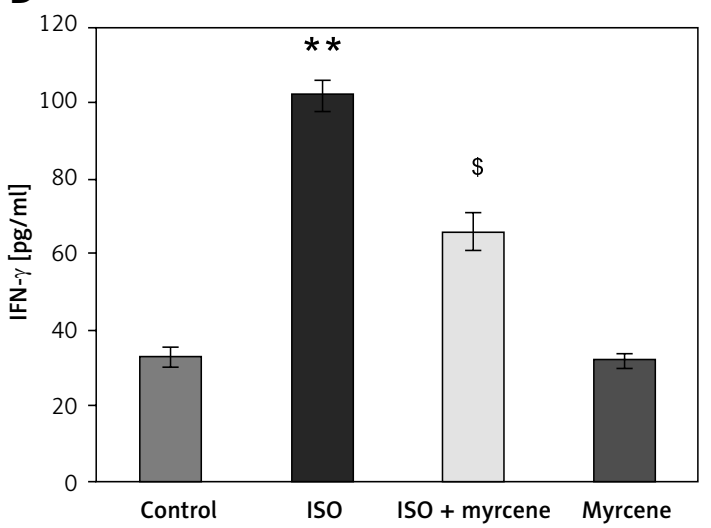

$\mathrm{F}$

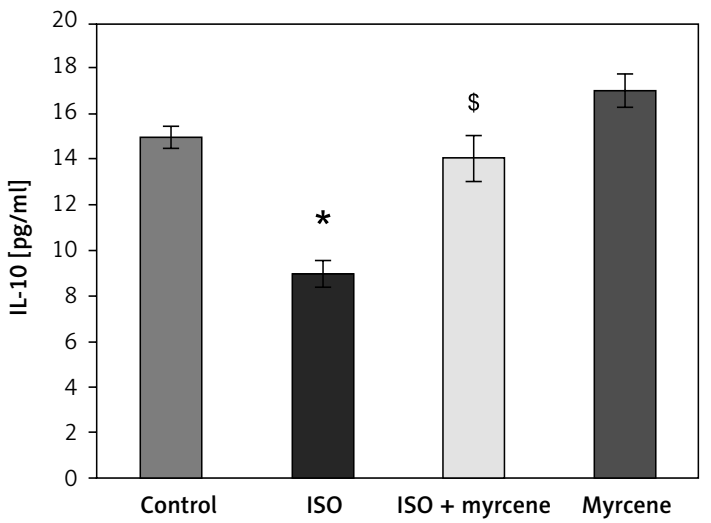

Figure 3. A-F represents the cytokines levels in the control and experimental group of rats. The detail of the experiment is given in the methodology section

Values are expressed as mean $\pm S E(n=12)$. Statistical significance expressed as ${ }^{*} p<0.05,{ }^{* *} p<0.01,{ }^{* * *} p<0.001$ compared to vehicle-treated controls, ${ }^{s} p<0.05,{ }^{\$ S} p<0.011 S O+$ myrcene compared to ISO-induced rats.

normal functioning of the heart, with its normal parameters disturbed and an increase in the expression of cardiac failure markers in the course of the malfunctioning of the heart, was demonstrated. Therefore, the present study was aimed at the potential therapeutic effects of myrcene in exerting its cardioprotection against ISO-induced cardiac hypertrophy in rats.

Isoproterenol induces heart failure by inhibiting systolic and diastolic function and causes myocardial infarction, which then leads to myocardial ischaemia, necrosis, and cardiac fibrosis [17]. The myocardial infarction inducing property of ISO is similar to myocardial infarction in humans and hence is used in animal models to study the cardioprotective properties of biomolecules; in our case, it was myrcene [18]. The present study elucidated the cardioprotective property of myrcene, in particular, protecting the myocardial functions and structure by modulating the inflammatory enzymes and the cardiac marker expression in the ISO-induced heart-failure animal model. 
A
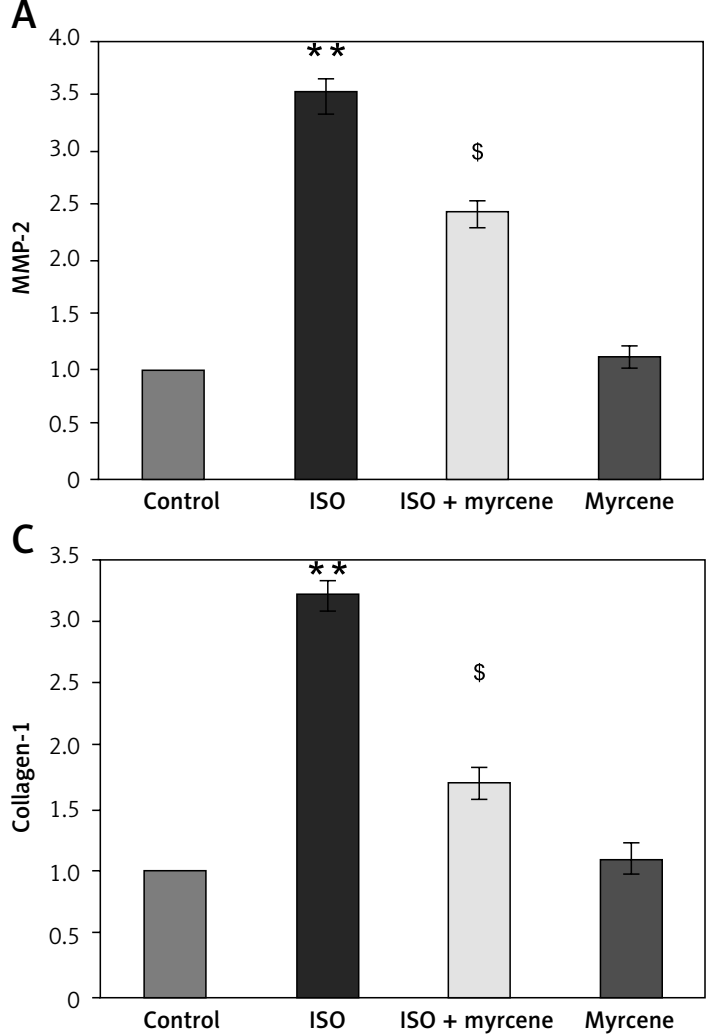

E

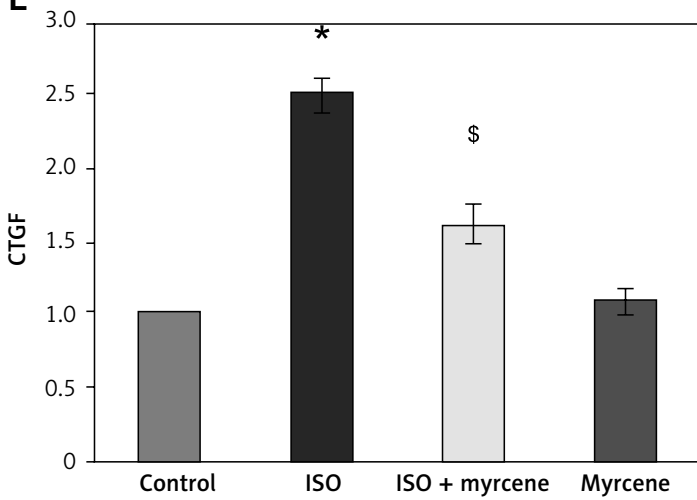

G

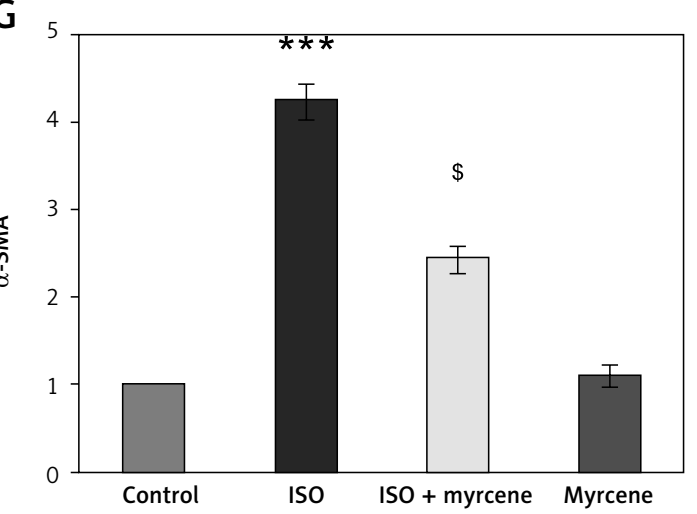

B

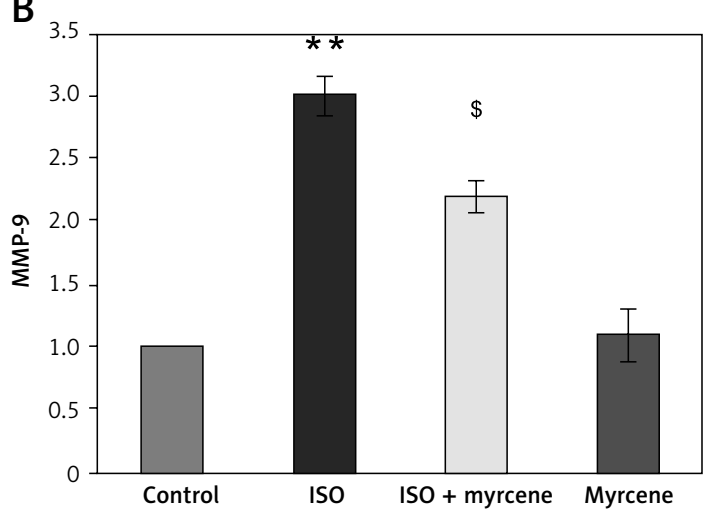

D

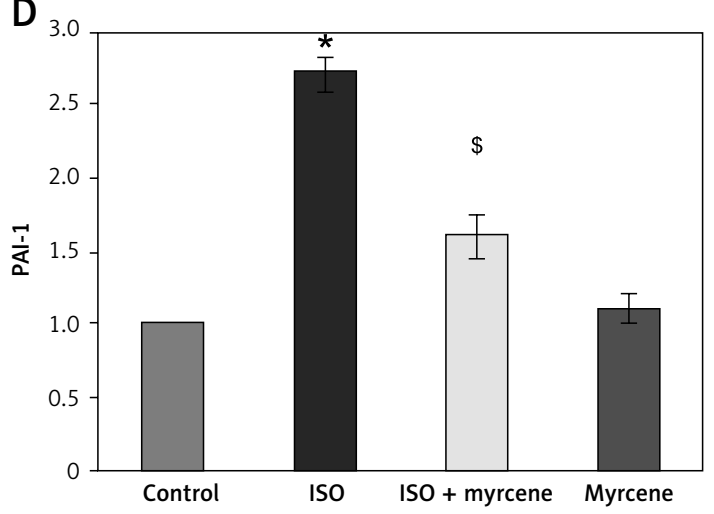

$\mathrm{F}$

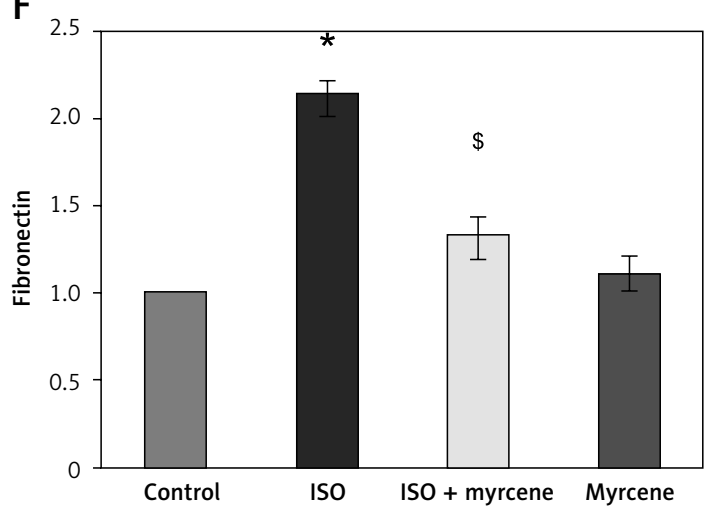

$\mathrm{H}_{3.5}$

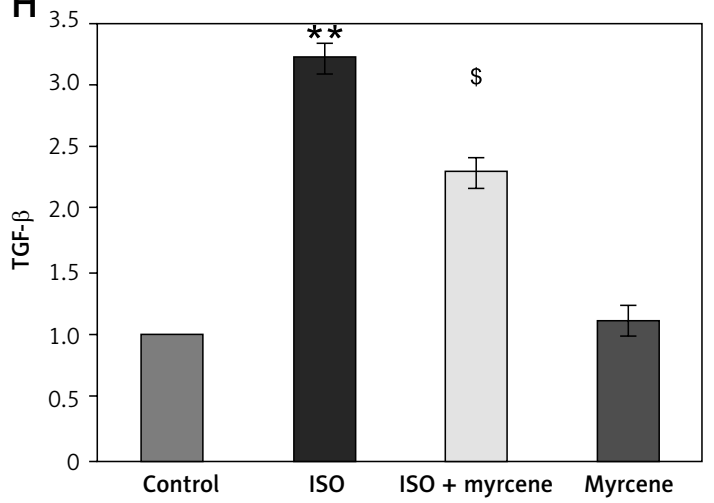

Figure 4. A-H represents GRT-PCR mRNA expression analysis of marker genes in the control and experimental groups of rats. The fold increase of gene expression is compared with the housekeeping gene GAPDH. The detail of the experiment is given in the methodology section

Values are expressed as mean $\pm S E(n=12)$. Statistical significance is expressed as ${ }^{*} p<0.05,{ }^{* *} p<0.01,{ }^{* * *} p<0.001$ compared to vehicle-treated controls, ${ }^{\$} p<0.05$ ISO + myrcene compared to ISO-induced rats. 
A

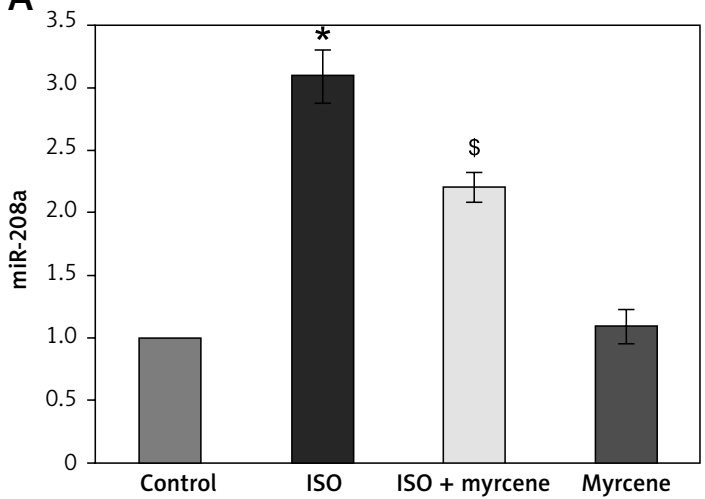

C

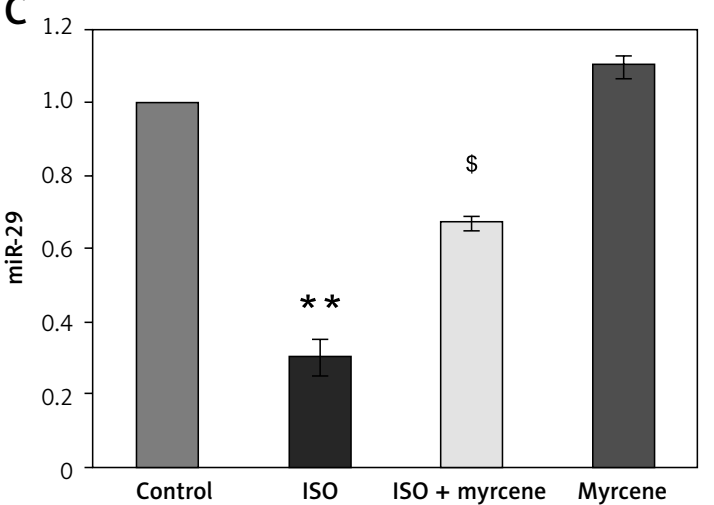

E

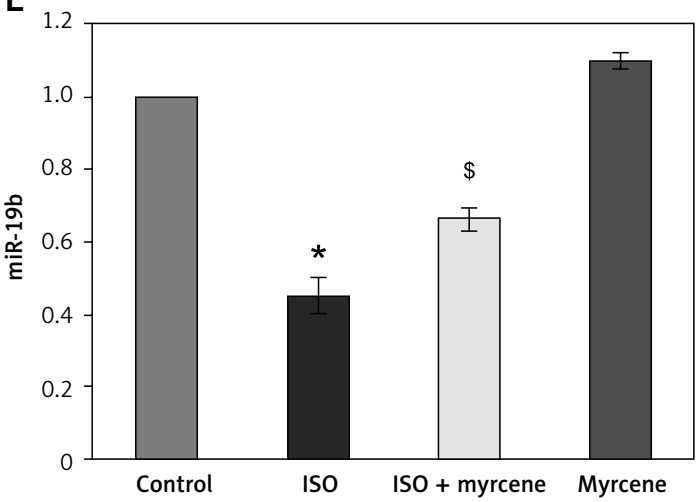

The Hw/Bw ratio is an indicator of cardiac hypertrophy [19], and it is due to the action of ISO that has significantly increased the heart rate [20] rather than the sham group for all weight levels. The increase in the Hw/Bw ratio was lessened when the rat groups were treated with myrcene, indicating that the effect of our candidate molecule on the cardiac muscles was significant. The degree of ventricular hypertrophy was estimated using the heart-to-body weight ratio $(\mathrm{Hw} / \mathrm{Bw})$ and was observed to be high in ISO-treated animals. The rise in the Hw/Bw ratio can be attributed to the increase in water content, infiltration of the inflammatory cells in response to the cardiac inju$r y$, and oedema in the cardio-muscular region [21]. This was further confirmed with the histological studies wherein inflammatory cell infiltrations,
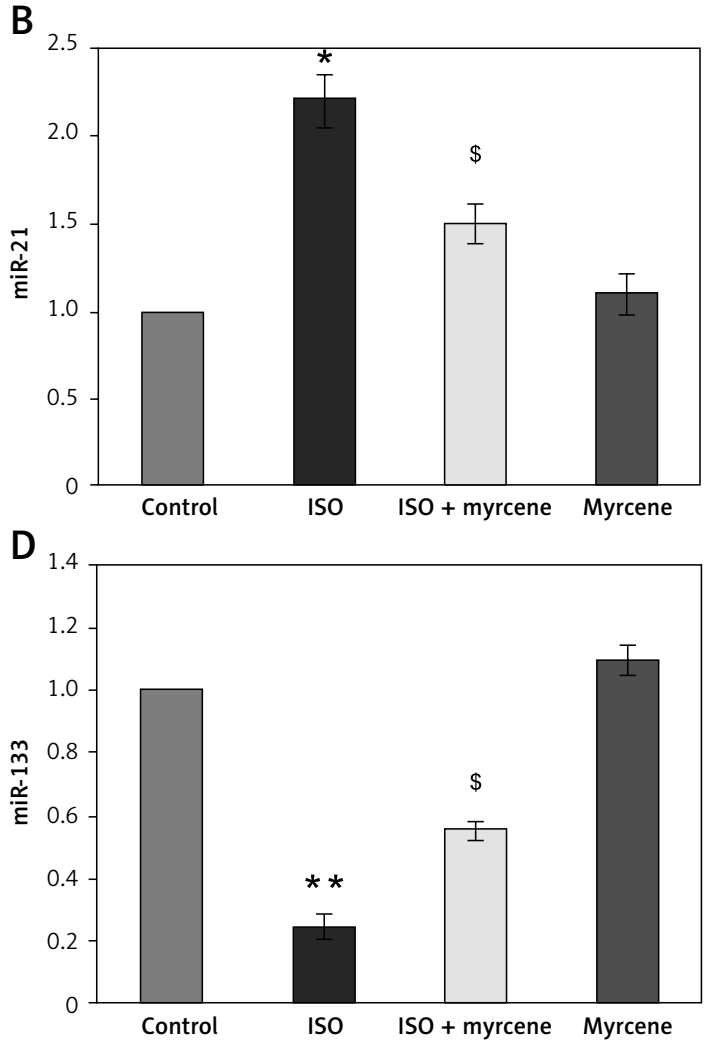

Figure 5. A-E represents expression analysis of microRNA such as miR-21, miR-208a, miR-29, miR$19 \mathrm{~b}$, and miR-133 in the control and experimental groups of rats. The detail of the experiment is given in the methodology section

Values are expressed as mean \pm S.E $(n=12)$. Statistical significance expressed as ${ }^{*} p<0.05,{ }^{* *} p<0.01$ compared to vehicle-treated controls, ${ }^{s} p<0.05150+$ myrcene compared to ISO-induced rats.

cardiac fibrosis, and thickening of the left ventricle of the myocardium were observed.

ISO increases the cardiac output and the heart rate in the animals $[22,23]$, and such increases can be curbed with myrcene treatment, which acts through the $\alpha 2$-adrenoceptor [24] and partially with its lipid-lowering activity [25], and reduces the heart rate and reverses the associated symptoms of hypertension and subsequent heart failure [26].

The levels of creatine kinase MB [27] and lactate dehydrogenase, which are important enzymes highly expressed during a heart attack by ISO [28] in response to cardiac permeability due to inflammation and myofibrillar disintegration [29] and are indicators of initial cardiac damage [30], were tested in the animals, and it was found 
A
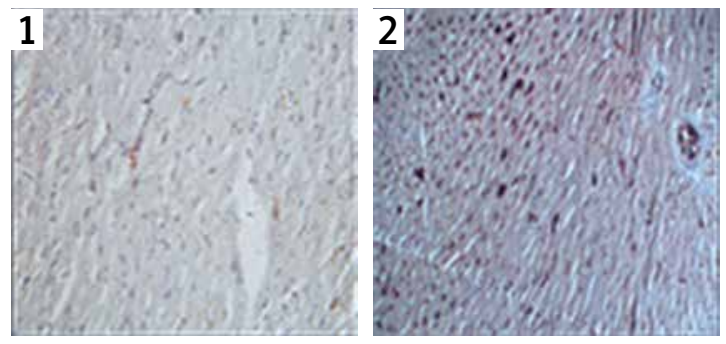

B

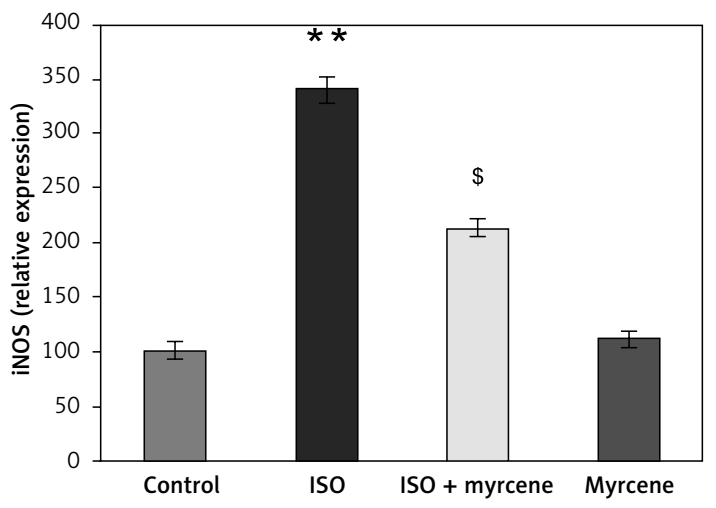

C
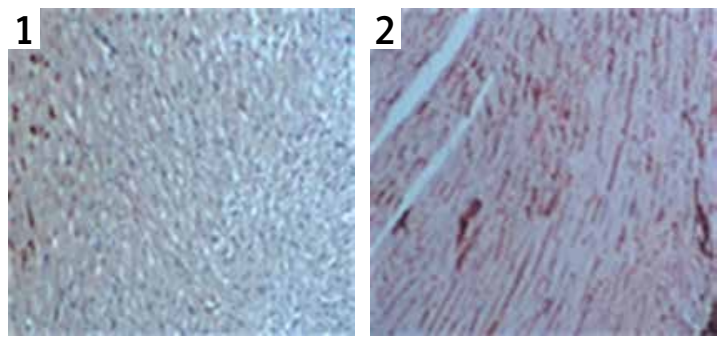

D

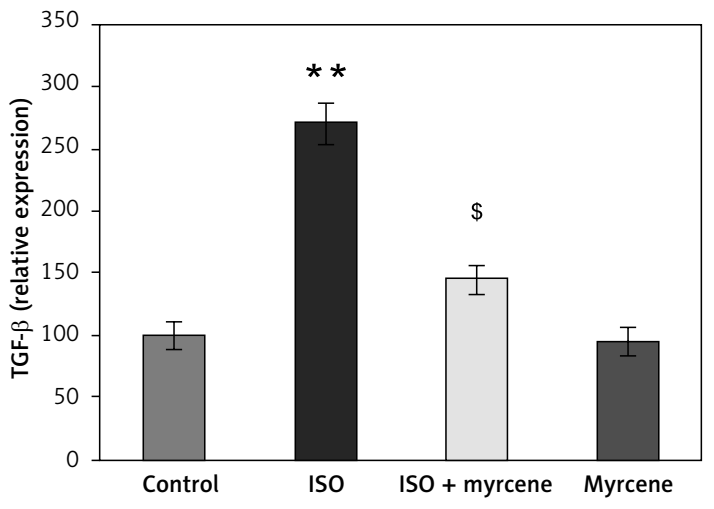

that the ISO-induced group had the highest levels whereas the myrcene-treated animals, which were earlier induced with ISO, had reduced expression levels, which may be due to its influence on the adrenoreceptor [24] that is responsible for the increased creatine kinase levels [31].

We observed abnormalities in the systolic-diastolic functions of the heart through electrocardiograph measurements, and they were used as a reference for the induction of myocardial infarc-
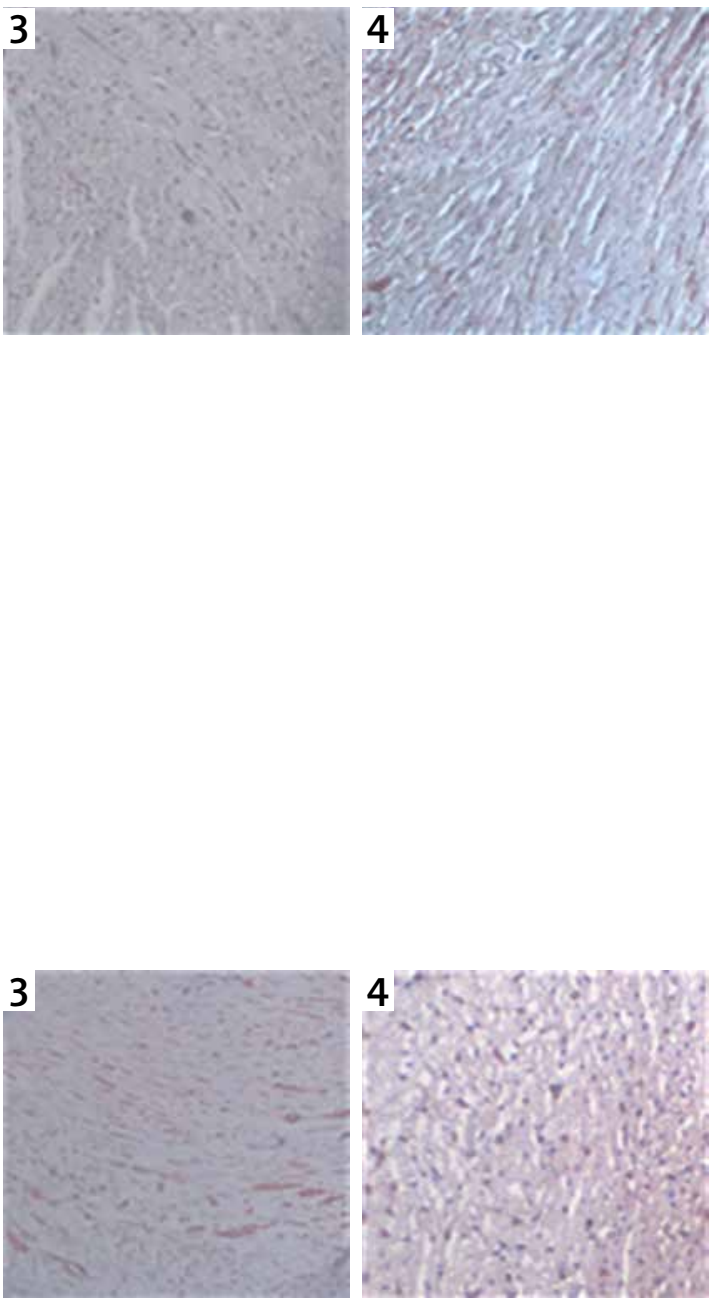

Figure 6. A-D represents the immunohistochemical $(\mathrm{IHC})$ analysis of iNOS and TGF- $\beta$ in the control and experimental groups of rats ( 1 - Control; 2 ISO; 3 - ISO + myrcene; 4 - Myrcene). The detail of the experiment is given in the methodology section

Values are expressed as mean $\pm S E(n=12)$. Statistical significance expressed as ${ }^{* *} p<0.01$ compared to vehicletreated controls, ${ }^{s} p<0.0515 O+$ myrcene compared to ISO-induced rats.

tion in ISO-induced animals [32]. They were represented in the values of LV end-diastolic diameter (LEVDd), left ventricular end-systolic diameter, and interventricular septal thickness in diastole (IVSTd), which showed increases due to cell membrane alterations [33], but which reduced to normal levels with the treatment of myrcene, which inhibited the ISO-induced ST-segment abnormalities, indicating the role played by myrcene in the protection of cardiac cell membranes [18]. 
Cardiac hypertrophy is associated with increased levels of IL-6 in its signalling pathway [34]. IL-6 is highly expressed in ISO-induced cardiac hypertrophy and is similarly recorded in our animals induced with ISO. This is the consistent and known observation of a close association between inflammation and cardiac hypertrophy in rats. However, due to the anti-inflammatory effect of myrcene, the levels of IL- 6 are reduced, which is a part of the protective property when used in higher concentration $[11,13]$. Similarly, TNF- $\alpha$ is highly expressed in ISO-induced heart failure animals [35] because it a major cytokine that has an effect on the contraction of cardiac muscle and contributes to myocardial injury [35]. These effects are hallmarks of cardiac injury in ISO-treated animals and an indicator of the infiltration of neutrophils and inflammation in the myocardial tissue and necrosis [21]. With the infiltration of mast cells, IL-4 secretion is higher in the ISO-induced cells, and they promote cardiac fibrosis (with partial participation from TGF- $\beta$ ), and an increase in the deposition of collagen is seen [36, 37], which impedes the cardiac rhythm [38]. Myrcene, with its anti-inflammatory property, reduces the expression of TNF- $\alpha, \mathrm{IL}-4$, and IFN- $\gamma$ [39] in the treated cells and helps in the recovery of myocardial tissues from injury and inflammation along with a simultaneous increase in the anti-inflammatory cytokine IL-10.

We then diversified our work towards elucidating the markers that are expressed in the advanced level of heart failure that is involved in the turnover of extracellular matrix and fibrosis of the cardiac muscles in the increased stiffness of the cardiac muscles and the resultant loss of myocardial elasticity, which are prime factors in the disruption of heart dysfunction [40]. The expression of these markers indicates the severity of the cardiac failure and the extent of extracellular matrix (ECM) degradation that it has undergone during cardiac remodelling in the course of the disease. The integrity of the ECM is governed by the complex interplay between matrix metalloproteinases and their tissue inhibitors (TIMPs). In the ISO-induced model animals, increased expression of MMP-2 and MMP-9 was observed. This is in line with previously observed results [41-43]. The induction of expression of the extracellular matrix genes is controlled by myrcene and thus results from the instability of the ECM matrix, which otherwise would result in degradation of the matrix protein and huge turnover [12].

As well as the biomarkers due to ECM turnover, we also studied the biomarkers that are circulating and are at the miRNA level. Our results are consistent with the existing data in which the expression of miR-208 [44] and miR-21 are high in animals with heart failure, and cardiac fibro- sis [17] and the trend was reversed in animals treated with myrcene, but the levels of expression of miR-29 [45], miR-133 [46], and miR-19b increased, indicating the damaged heart is on the path to recovery even at the molecular level, and myrcene has effectively mediated it. These circulating markers are easier to detect, and diagnosis based on them would give advance information on the probable risks, and therapies based on antisense technology would help in designing sustained and potent silencing of miRNA implicated in heart failure.

The pathogenesis of resultant injury on the cardiac muscles due to ISO would be due to the generation of nitric oxide, aided by the in creased expression of iNOS in the heart tissues and in the activation of signalling by TGF- $\beta$ to wards pro-fibrosis and are important and necessary for the induction of the cardiac fibroblast towards fibrosis. Control of the injury through the reduction of iNOS and TGF- $\beta$ expression is aided by myrcene $[12,13]$, which increases the cardioprotection.

In conclusion, we present our results together to demonstrate and support that myrcene suppresses MMP-2 and MMP-9 expression and regulates the expression of iNOS, TGF- $\beta$, and miRNA, which are profibrotic. It also decreases the ECM turnover in the pathology of cardiac failure in a rat model. The present study highlights the use of myrcene in the treatment of cardiac failure by utilising its anti-inflammatory properties, and may support future therapies for cardiac failure.

\section{Conflict of interest}

The authors declare no conflict of interest.

\section{References}

1. Nieminen MS, Bohm M, Cowie MR, Drexler H, Filippatos GS, Jondeau G. Guideline ESCCFP Executive summary of the guidelines on the diagnosis and treatment of acute heart failure: the task force on acute heart failure of the European Society of Cardiology. Eur Heart J 2005; 26: 384-416.

2. Zhang Z, Wang B, Fei A. BDNF contributes to the skeletal muscle anti-atrophic effect of exercise training through AMPK-PGC1alpha signaling in heart failure mice. Arch Med Sci 2019; 15: 214-22.

3. Omland T. Advances in congestive heart failure management in the intensive care unit: B-type natriuretic peptides in evaluation of acute heart failure. Crit Care Med 2008; 36 (1 Suppl): S17-27.

4. Talman V, Ruskoaho H. Cardiac fibrosis in myocardial infarction-from repair and remodeling to regeneration. Cell Tissue Res 2016; 365: 563-81.

5. Michalska-Kasiczak M, Bielecka-Dabrowa A, von Haehling S, Anker S, Rysz J, Banach M. Biomarkers, myocardial fibrosis and co-morbidities in heart failure with preserved ejection fraction: an overview. Arch Med Sci 2018; 14: 890-909. 
6. Lin $Y H$, Lin LY, Wu YW, et al. The relationship between serum galectin-3 and serum markers of cardiac extracellular matrix turnover in heart failure patients. Clin Chim Acta 2009; 409: 96-9.

7. Lombardi R, Betocchi S, Losi MA, et al. Myocardial collagen turnover in hypertrophic cardiomyopathy. Circulation 2003; 108: 1455-60.

8. Ma Y, de Castro Bras LE, Toba H, et al. Myofibroblasts and the extracellular matrix network in post-myocardia infarction cardiac remodeling. Pflugers Arch 2014; 466: 1113-27.

9. Katz AM, Zile MR. New molecular mechanism in diastolic heart failure. Circulation 2006; 113: 1922-5.

10. Mocan M, Mocan Hognogi LD, Anton FP, et al. Biomarkers of inflammation in left ventricular diastolic dysfunction. Dis Markers 2019; 2019: 7583690.

11. de Cassia da Silveira e Sa R, Andrade LN, de Sousa DP. A review on anti-inflammatory activity of monoterpenes. Molecules 2013; 18: 1227-54.

12. Rufino AT, Ribeiro M, Sousa C, et al. Evaluation of the anti-inflammatory, anti-catabolic and pro-anabolic effects of E-caryophyllene, myrcene and limonene in a cell model of osteoarthritis. Eur J Pharmacol 2015; 750: 141-50.

13. Hwang E, Ngo HTT, Park B, Seo SA, Yang JE, Yi TH. Myrcene, an aromatic volatile compound, ameliorates human skin extrinsic aging via regulation of MMPs production. Am J Chin Med 2017; 45: 1113-24.

14. Subramanian U, Kumar P, Mani I, et al. Retinoic acid and sodium butyrate suppress the cardiac expression of hypertrophic markers and proinflammatory mediators in Npr1 gene-disrupted haplotype mice. Physiol Genomics 2016; 48: 477-90.

15. Umadevi S, Gopi V, Elangovan V. Regulatory mechanism of gallic acid against advanced glycation end products induced cardiac remodeling in experimental rats. Chem Biol Interact 2014; 208: 28-36.

16. Molojavyi A, Lindecke A, Raupach A, Moellendorf S, Kohrer K, Godecke A. Myoglobin-deficient mice activate a distinct cardiac gene expression program in response to isoproterenol-induced hypertrophy. Physiol Genomics 2010; 41: 137-45

17. Li L, Zhao Q, Kong W. Extracellular matrix remodeling and cardiac fibrosis. Matrix Biol 2018; 68-69: 490-506.

18. Zhou R, Xu Q, Zheng P, Yan L, Zheng J, Dai G. Cardioprotective effect of fluvastatin on isoproterenol-induced myocardial infarction in rat. Eur J Pharmacol 2008; 586 244-50.

19. Sun JM, Wang CM, Guo Z, et al. Reduction of isoproterenol-induced cardiac hypertrophy and modulation of myocardial connexin43 by a KATP channel agonist. Mol Med Rep 2015; 11: 1845-50.

20. Zahabi A, Picard S, Fortin N, Reudelhuber TL, Deschepper CF. Expression of constitutively active guanylate cyclase in cardiomyocytes inhibits the hypertrophic ef fects of isoproterenol and aortic constriction on mouse hearts. J Biol Chem 2003; 278: 47694-9.

21. Patel V, Upaganlawar A, Zalawadia R, Balaraman R. Cardioprotective effect of melatonin against isoproterenol induced myocardial infarction in rats: a biochemical, electrocardiographic and histoarchitectural evaluation. Eur J Pharmacol 2010; 644: 160-8.

22. Cihak R, Kolar F, Pelouch V, Prochazka J, Ostadal B, Wi dimsky J. Functional changes in the right and left ventricle during development of cardiac hypertrophy and after its regression. Cardiovasc Res 1992; 26: 845-50.

23. Tang L, Taylor PB. Altered contractile function in iso proterenol-induced hypertrophied rat heart. J Hypertens 1996; 14: 751-7.
24. Rao VS, Menezes AM, Viana GS. Effect of myrcene on nociception in mice. J Pharm Pharmacol 1990; 42: 877-8.

25. Lee H, Woo M, Kim M, Noh JS, Song, YO. Antioxidative and cholesterol-lowering effects of lemon essential oil in hypercholesterolemia-induced rabbits. Prev Nutr Food Sci 2018; 23: 8-14.

26. Gavras I, Manolis AJ, Gavras H. The alpha2-adrenergic receptors in hypertension and heart failure: experimental and clinical studies. J Hypertens 2001; 19: 2115-24.

27. Sax H, Contesse J, Dubach P, Reinhart WH. Creatine kinase $M B$ during myocardial infarction: relationship to preexisting coronary heart disease and medication. Acta Cardiol 1997; 52: 423-30.

28. Adams JE $3^{\text {rd }}$, Bodor GS, Dávila-Román VG, et al. Cardiac troponin I. A marker with high specificity for cardiac injury. Circulation 1993; 88: 101-6.

29. Apple FS. Tissue specificity of cardiac troponin I, cardiac troponin T and creatine kinase-MB. Clin Chim Acta 1999; 284: 151-9.

30. Preus M, Bhargava AS, Khater AE, Gunzel P. Diagnostic value of serum creatine kinase and lactate dehydrogenase isoenzyme determinations for monitoring early cardiac damage in rats. Toxicol Lett 1988; 42: 225-33.

31. Jingu $Y$, Aoki $Y$, Itoh $H$, Saruta T. Serum creatine kinase release and alpha- and beta-adrenergic receptors. Arch Int Pharmacodyn Ther 1986; 282: 262-75.

32. Rajadurai M, Prince PS. Preventive effect of naringin on isoproterenol-induced cardiotoxicity in Wistar rats: an in vivo and in vitro study. Toxicology 2007; 232: 216-25.

33. Holland RP, Brooks H. TQ-ST segment mapping: critical review and analysis of current concepts. Am J Cardiol 1977; 40: 110-29.

34. Al-Rasheed NM, Al-Oteibi MM, Al-Manee RZ, et al. Simvastatin prevents isoproterenol-induced cardiac hypertrophy through modulation of the JAK/STAT pathway. Drug Des Devel Ther 2015; 9: 3217-29.

35. Ramani R, Mathier M, Wang P, et al. Inhibition of tumor necrosis factor receptor-1-mediated pathways has beneficial effects in a murine model of postischemic remodeling. Am J Physiol Heart Circ Physiol 2004; 287: H1369-77.

36. Burton OT, Darling AR, Zhou JS, et al. Direct effects of IL-4 on mast cells drive their intestinal expansion and increase susceptibility to anaphylaxis in a murine model of food allergy. Mucosal Immunol 2013; 6: 740-50.

37. Peng H, Sarwar Z, Yang XP, et al. Profibrotic role for interleukin-4 in cardiac remodeling and dysfunction. Hypertension 2015; 66: 582-9.

38. Brower GL, Gardner JD, Forman MF, et al. The relationship between myocardial extracellular matrix remodeling and ventricular function. Eur J Cardiothorac Surg 2006; 30: 604-10.

39. Souza MC, Siani AC, Ramos MF, Menezes-de-Lima OJ, Henriques MG. Evaluation of anti-inflammatory activity of essential oils from two Asteraceae species. Pharmazie 2003; 58: 582-6.

40. Westermann D, Lindner D, Kasner $M$, et al. Cardiac inflammation contributes to changes in the extracellular matrix in patients with heart failure and normal ejection fraction. Circ Heart Fail 2011; 4: 44-52.

41. Kato TS, Chokshi A, Singh P, et al. Markers of extracellular matrix turnover and the development of right ventricular failure after ventricular assist device implantation in patients with advanced heart failure. J Heart Lung Transplant 2012; 31: 37-45.

42. Spinale FG. Myocardial matrix remodeling and the matrix metalloproteinases: influence on cardiac form and function. Physiol Rev 2007; 87: 1285-42. 
43. Yang YJ, Zhang X, Zhu WL, Huang Y. Involvement of matrix metalloproteinases in the regulation of myocardial extracellular matrix in patients with congestive heart failure. Zhonghua Yi Xue Za Zhi 2006; 86: 1693-6.

44. Montgomery RL, Hullinger TG, Semus HM, et al. Therapeutic inhibition of miR-208a improves cardiac function and survival during heart failure. Circulation 2011; 124: 1537-47.

45. Lew WY, Bayna E, Dalle Molle E, Contu R, Condorelli G, Tang T. Myocardial fibrosis induced by exposure to subclinical lipopolysaccharide is associated with decreased miR-29c and enhanced NOX2 expression in mice. PLoS One 2014; 9: e107556.

46. Sucharov C, Bristow MR, Port JD. miRNA expression in the failing human heart: functional correlates. J Mol Cell Cardiol 2008; 45: 185-92 